\title{
LA INVERSA GENERALIZADA
}

RODOLFO BAEZA VEGA

Sea $F_{m, n}$ el conjunto de las matrices $m x$ con en tradas en $F$. Consideremos $A \varepsilon F_{m, n}$ y $B \varepsilon F_{m, 1}$. Debemos encontrar $X \in F_{n, 1}$ tal que $A X=B$. Es decir buscamos las solu ciones del sistema lineal de m ecuaciones con $n$ incognitas

$$
\mathrm{AX}=\mathrm{B} \cdot \cdot \cdot \cdot \cdot(*)
$$

Observemos que :

1) Si $\rho(A)=\rho(A \mid B)=m=n$ entonces (*) tiene solución única y está dada por

$$
X=A^{-1} \quad B
$$

donde $\mathrm{A}^{-1}$ es 1 a inversa multiplicativa de $\mathrm{A}$.

2) $\operatorname{si} \rho(A)=\rho(A \mid B)=n \neq m$ entonces existen $\infty$ soluciones que tambien queremos escribirlas como $X=A_{1} B$.

3) Si $\rho(A) \neq \rho(A \mid B)$ no existe solución pero nos gustaria encontrar algun $X$ que haga "minima" la diferencia AX-B. En caso que hubiera varios de tales $X$ nos interesa en 
contrar aquellas que sean mas "pequeñas" (naturalmente, debe mos hablar de algún tipo de mêtrica en las matrices). Tales $X$ tambien queremos escribirlas de la forma $X=A_{1} B$.

$$
\text { Las matrices } A_{1} \text { que sirven para 2) y 3) tienen }
$$

algunas propiedades parecidas a las de la inversa de A. Por tal razón se les llama de alguna manera como inversas de A, por ejemplo "Inversa generalizada", "seuda inversa". "inversa de Penrose" o "Inversa de Moore-Penrose".

El objetivo de estas notas es ilustrar su cálcu lo y aplicación en el análisis de los sistemas de ecuacio nes lineales. Tal analisis puede hacerse, sin aumentar Ios problemas, si $B \varepsilon F_{m, k} \circ$ sea si se trata de un conjunto de sistemas de ecuaciones 1 ineales de $m$ ecuaciones $y$ n incógnitas cada uno. En lo que sigue A* representa la traspuesta de A ( $i$ $A(s i F=$ C).

\section{Definición :}

Sea $A \varepsilon F_{m, n}$ y $G \in F_{n, m}$ decimos que

1) $G$ es (1) inversa de $A$ si $A G A=A$

2) $G$ es (2) inversa de $A$ si $G A G=G$

3) $G$ es (3) inversa de $A$ si ( $A G) *=A G$

4) $G$ es (4) inversa de $A s i(G A) *=G A$

tal que $G$ es (1) inversa de A.

$$
\text { 1.1. Proposiciôn : } A \in F_{m, n} \exists G \varepsilon F_{n, m}
$$

invertibles tal que

$$
\text { Demostración : } \operatorname{sean} P \varepsilon_{m, m} \text { y } Q \varepsilon_{n, n}
$$

$$
P A Q=D=\left(\begin{array}{c|c}
I_{r} & 0 \\
\hline 0 & 0
\end{array}\right)=\text { forma canónica de }
$$

Ia equivalencia de matrices. La matriz $G=Q D^{t} P$ es (1) inver sa de A.

$$
\text { 1.2. Proposición 2 (*) tiene solución } \Longleftrightarrow
$$

$A G B=B, \forall G$ (1) inversa de A. 
versa de A entonces:

Demostración: Sea $A X=B$ y sea $G$ una (1) in$A G B=A G(A X)=(A G A) X=A X=B$. Si $A G B=B$ enton ces $X=G B$ es solución de $(*)$

1.3.Proposición 3 : Si (*) tiene solución entonces $X=G B+(I-\overline{G A}) H$ es la solución general de (*) para $G$ (1) inversa de $A$.

Demostración : Es claro que $G B+(I-G A) H$ es solución de $(*)$. Si $X$ es soluciôn de $(*)$ entonces haciendo $H=(I-G A) X$ tenemos que $X=G B+(I-G A) H$

Las tres proposiciones anteriores resuelven las partes (1) y (2) iniciales. Para analizar el problema de los sistemas inconsistentes utilizaremos la norma de una ma$\operatorname{triz} A=\left(a_{i j}\right) \varepsilon F_{m, n}$ definida por

$$
\|A\|^{2}=\sum_{i, j} \quad\left|a_{i j}\right|^{2}=\operatorname{tr}(A * A)
$$

\section{Definición:}

1) Se dice que $X$ es una solución mínima cuadrá. tica de (*) si $\|A X-B\|$ es minimal

2) La ecuación normal de $(*)$ es $A * A X=A * B \ldots(* *)$

\subsection{Proposición 1:}

1) (xis:) siempre tiene solución

2) X es solución minimo cuadrática de

$* \Leftrightarrow x$ es solución de $(* *)$

Demostración: Sea $G$ una (1) inversa de $A * A$ basta probar que $A * A G A * B=A * B$ (por Proposición 1.2)

$$
\text { Como } A * A G A * A=A * A \text { entonces }(A * A G-I) A * A=\cdots \text {, }
$$

'sego $(A * A G-I) A * A(A * A G-I) *=0$ De ahi tenemos que

$[(A * A G-I) A *][(A * A G-I) A *] *=0$ y en consecuencia

$\left.\|(A * A G-I) A *\|\right|^{2}=0$ de donde $(A * A G-I) A *=0$. 


\section{Luego $A * A G A *=A *$ y por lo tanto $A * A G A * B=A * B$.}

\subsection{Observaciones:}

1) $G$ es (1.3) inversa de $A \Leftrightarrow A * A G=A *$

2) $S i$ es (1.3) inversa de A entonces

$X=G B$ es solución mínimo cuadrática de (*). Por esta razón la (1.3) inversas son llamadas Inversas-minimo-cuadráticas. Nótese que las inversas minimo cuadráticas de A son justamen te una solución mínimo cuadrática de $A X=I$.

$G=H A *$ es $(1,2,3)$ inversa de $A$.

3) Si $\mathrm{H}$ es una (1) inversa de $A * A$ entonces Demostraciones :

1) $\Rightarrow \quad A * A G=A *(A G)=A *(A G) *=(A G A) *=A *$

\&) Como $(A G A) *=A * G * A *=(A * A G) G *(A * A G)=$

entonces $G$ es (1) inversa de $A$.

es (3) inversa de A.

Como $(A G) * G * A *=G * A * A G=A G$ entonces $G$

2) Siendo $G$ (1.3) inversa de A tenemos que $A * A G=A *$ luego $A * A G B=A * B \quad \therefore X=G B$ es solución de $(* *)$ y en consecuancia es solución mínimo cuadrática de $(*)$.

3) Como $H$ es (1) inversa de $A * A$ entonces $A * A H A * A=A * A \circ$ equivalentemente: $A * A H * A * A=A * A$ luego $A * A(H A * A-H * A * A)=0$ y por el argumento utilizado en la proposición (2.1.(a)) tenemos que AHA* $A=A H * A * A$.

De modo análogo vemos que

$A * A H * A * A=A * A \Rightarrow A * A(H * A * A-I)=0$ de donde $A(H * A * A-I)=0$, sea $A H * A * A=A \circ$ sea $A H A * A=A \therefore A G A=A$.

Además $G A G=H A * A H A *=H(A H * A * A) *=H A *=G$

Finalmente : $A H A * A=A H * A * A \Rightarrow(A H-A H *) A * A=0$

de donde $(A H-A H *) A *=0$ ○ sea $A H A *=A H * A *$, luego $(A G) *=(A H A *) *(A H * A *) *=A H A *=A G$. 
3. Definición :

Sea $x$ una solución de $(*)$. Diremos que $x$ es una solución de norma mínima si

$$
\|x\|^{2} \text { es minimal. }
$$

Entonces :

$$
3.1 \text { Proposición : Sea } X \text { una solución de }(*)
$$

$(\mathrm{AH}=0 \Rightarrow \mathrm{X} * \mathrm{H}=0$ es solución de norma minima de $(*)$ :

\subsection{Observaciones:}

1) $G$ es (1.4) Inversa de $A \Longleftrightarrow A A * B_{*} *=A$

2) Si $G$ es (1.4) Inversa de $A v(*)$ lene solución, entonces $x=G B$ es una solución de norma minima.

Demostraciones:

$$
\text { 1) } \begin{aligned}
\Rightarrow & A A * G *=A(G A) *=A G A=A \\
\Leftrightarrow) & A G A=A G(A *) *=A G(G A A *) * A G A A * A * \\
& =A(G A A *) G *=A A * G *=A \text { y ademas: } \\
& (G A) *=A * G *=(G A A *) *=G(A A * G *)=G A
\end{aligned}
$$

2) Como $G$ es (1) inversa de $A$ y $(*)$ tiene solución entonces $X=G B$ es una solución de $(*)$. Además
$A H=0$ entonces $X * H=(H * X) *=(H * G b) *=[H * G(A G b)] *=\left[H *(G A) G b_{-}^{*} *=\right.$ $=[H * A * G * G b] * b * G * G A H=b * G * G \cdot 0=0$

ción 3.1 se tiene que $X=G b$ es solución de norma minima.

\section{Definición :}

Una mejor solución de $(*)$ es una solución mîn ma cuadrâtica de norma minima.

lución de (*)

$$
\text { 4.1. Proposición : Existe una única meior so }
$$


Demostraciôn: La existencia se debe a la prososición 2.1 y paraver la unicidad, supongamos que $X e^{Y}$ sean soluciones de $(* *)$ entonces $: A * A X=A * A Y=A * b$ y de aqui obtenemos que $A * A(X-Y)=0$. Premultiplicando esta úl tima igualdad por $(X-Y) *$, obtenemos que $\|A(X-Y)\|=0 \operatorname{co}=$ mo $\|A\| \neq 0$, tenemos que $\|X-Y\|=0$ y finalmente que

\subsection{Observaciones:}

$(1,2,3,4)$ inversa de A.

2) Si $G$ es una $(1,2,3,4)$ inversa de $A$, en tonces ella es unica $y$ es la mejor solución de $A X=I$.

\section{Demostraciones:}

1) Por ser G la mejor solución de $A X=I$, teremos que $A * A G=A *$ y por la observación 2.1 concluimos que $G$ es $(1,3)$ inversa de $A$.

Como $G$ es 1 -inversa de $A$, entonces $A * A(G A-I)_{1}=0$ y ya que $G$ es solución de norma mínima de niendo que $G *(G A-I)=0$ o sea $G * G A=G *$.

Con esta ulltima igualdad es inmediato probar que $G$ es $(2.4)$ inversa de $A$, en efecto : $(G A) *=A * G *=A *(G * G A)=(G * G A) * A=G A$ y $G A G=(G A) G=$ $=(G A) * G=(G * G A) *=G$

2) Basta probar que $G$ es la mejor solución de $A X=I$, pues en este caso la proposición 4.1. garantiza la unicidad. G es solución de $A^{*} A X=A *$ porque $A * A G=A *(A G)=A *(A G) *=(A G A) *=A *$. Ademas si $A * A H=0$ entonces $G * H=(G A G) * H=G *$. (GA)*H=G*GAH=G* (GAG) $A H=$ $=G * G(A G) A H=G * G(A G) * A H=G * G G * A * A H=G G G * 0=0 y$ por la proposición 3.1 se tiene que $X$ es solución de norma mi
nima.

\section{Definiciôn:}

La Unica $(1,2,3,4)$ inversa de A es llamada la MOORE-PENROSE inversa de A y se designa por: A 
es $x=A^{+} B$

A entonces por observación : Como $A^{+}$es $(1,3)$ inversa de A $x=A^{+} B$ es solución mînimo cuadrática de (*). Además por ser A una $(2,3,4)$ inversa de $A$ tenemos que

$\left(A * A^{+}\left(A^{+}\right) * A^{+}\right) *=\left(A^{+}\right) *$. Luego para cualquier $H$ tal que $A * A H=0$ es cierto que $\left(A^{+} B\right) * H=0$ ya que $\left(A^{+} B\right) * H=$ $=B *\left(A^{+}\right) * H=B *\left(A^{+}\right) * A^{+}\left(A^{+}\right) * A * A H=B *\left(A^{+}\right) * A^{+}\left(A^{+}\right) \cdot 0=0$ En consecuencia, por proposición 3.1

$A^{+} B$ es una solución de norma minima de $(* *)$

5.2. Observación : Para completar el estudio propuesto solo falta la metodologia que nos permita calcular $\mathrm{A}^{+}$- Para ello disponemos de las siguientes técnicas:

entonces

1) Si A es una matriz de rango completo

$$
A^{+}=(A * A)^{-1} A * \text { si } o(A)=n \text { y } A^{+}=A *(A A *)^{-1}
$$

si $\rho(A)=m$

el menor entero tal que $a_{r} \neq 0$ donde $\begin{aligned} & 2) \\ & S_{i} \text { es deficiente, }\end{aligned}$

$$
\sum_{i=1}^{m} a_{i} x^{i}
$$

es el polinomio caracteristico de B. Sea

$$
\begin{gathered}
T=-\frac{1}{a_{r}}\left[a_{r+1} I+\ldots .+a_{m} B^{m-r-1}\right] \text { entonces } \\
A^{+}=A * T .
\end{gathered}
$$

$G$ es $(1,2,3,4)$ Demostraciones: En general para probar que $G$ es $(1,2,3,4)$ inversa de A vicisa probar que G satisface las igualdades $A * A G=A *$ (observación 2.2.1.) y $G * G A=G *$ (Demostración de la ubservación 4.2.1.). 
1) Si $\rho(A)=n$ entonces $A * A$ es invertible y si $G=(A * A)^{-1} A *$, entonces: $A * A G=A *$ y $G A=I_{n}$ la demostración es anâloga. Luego $G * G A=G *$. En caso que $\rho(A)=m$,

2) Basta probar que $A * A A * T=A *$ porque ha ciendo $G=A * T$ tenemos que $A * A G=A *$ y en consecuencia $G$ es $(1,3)$ inversa de $A$. Como $G$ es 1 - Inversa de A entonces $G * G A=T * A G A=T * A=G *$

Por teorema de Cayley Hamilton tenemos que $a_{r} B^{r}+\ldots+a_{m} B^{m}=0$ luego $B^{r}=B^{r+1} T$ o sea $A A *\left[(A A *)^{r-1}-(A A *)^{r} T\right]=0$. Empleando el mismos arg $\underline{u}$ mento usado en la demostraciôn de la proposición 2.1 obtene mos que

$$
\begin{aligned}
& A *\left[(A A *)^{r-1}-(A A *)^{r} T\right]=0 \text { es decir } \\
& A * A \quad\left[(A * A)^{r-2} A *-(A * A)^{r-1} A * T\right]=0 \text { y, en ecnsecuencia } \\
& A\left[(A * A)^{r-2} A *-(A * A)^{r-1} A * T\right]=0 \text {, Hemos probado que } \\
& B^{r-1}=B^{r} T \text {. De ese modo es posible llegar a } B=B^{2} T \text { es } \\
& \text { decir } A A * A A * T=A A * \text { de donde } A * A A^{*} T=A * \text {. }
\end{aligned}
$$

